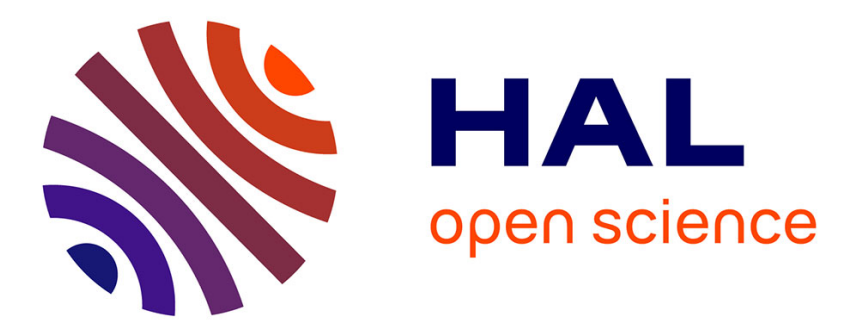

\title{
Improved Set-Membership Estimation Approach based on Zonotopes and Ellipsoids
}

\author{
Sofiane Ben Chabane, Cristina Stoica Maniu, Teodoro Alamo, Eduardo \\ Fernandez Camacho, Didier Dumur
}

\section{- To cite this version:}

Sofiane Ben Chabane, Cristina Stoica Maniu, Teodoro Alamo, Eduardo Fernandez Camacho, Didier Dumur. Improved Set-Membership Estimation Approach based on Zonotopes and Ellipsoids. 2014 European Control Conference (ECC), Jun 2014, Strasbourg, France. 10.1109/ECC.2014.6862412 . hal-00973454

\section{HAL Id: hal-00973454 \\ https://hal-centralesupelec.archives-ouvertes.fr/hal-00973454}

Submitted on 16 Mar 2020

HAL is a multi-disciplinary open access archive for the deposit and dissemination of scientific research documents, whether they are published or not. The documents may come from teaching and research institutions in France or abroad, or from public or private research centers.
L'archive ouverte pluridisciplinaire HAL, est destinée au dépôt et à la diffusion de documents scientifiques de niveau recherche, publiés ou non, émanant des établissements d'enseignement et de recherche français ou étrangers, des laboratoires publics ou privés. 


\title{
Improved Set-Membership Estimation Approach based on Zonotopes and Ellipsoids
}

\author{
S. Ben Chabane, C. Stoica Maniu, T. Alamo, E.F. Camacho, D. Dumur
}

\begin{abstract}
This paper presents an improved approach for guaranteed state estimation combining set-membership estimation techniques based on zonotopes and ellipsoids, applied on linear discrete-time systems with unknown but bounded perturbations and noises. The proposed approach starts with a zonotopic approximation and continues with an ellipsoidal approximation; this allows to manage the trade-off between the accuracy of the zonotopic estimation and the reduced complexity of the ellipsoidal estimation. A new criterion based on the $P$-radius of a zonotope is proposed to make the transition from the zonotopic estimation to the ellipsoidal estimation. An illustrative example is analyzed to show the advantages of the proposed approach.
\end{abstract}

\section{INTRODUCTION}

More often, the state estimation problems are solved by implementing a stochastic approach based on a probabilistic description of the perturbations and measurement noises [1]. This requires to assume that the individual perturbations are realizations of random variables characterized statistically by their average, covariance, probability density etc. However, sometimes the probabilistic assumptions are difficult to verify. Thus, it may be more realistic to assume that the errors belong to compact bounded sets. This corresponds to the deterministic approach or the set-membership estimation [2], [3], [4], [5], where perturbations are considered unknown but bounded. The set-membership estimation method leads to an estimation set containing the state vector. While their use was severely restricted in the $80 \mathrm{~s}$ due to the low capacity of available computers, these approaches have been widely used over the last two decades by many researchers [6], [7], [8], [9], [10].

There are several sets used to implement set-membership estimation techniques: parallelotopes [8], [9], ellipsoids [2], [6], [10], [11], [12], zonotopes [13], [14], [15], [16].

Ellipsoids have been used by different authors [11], [12], [17] due the simplicity of formulation. To minimize the size of the ellipsoidal estimation, two criteria are mainly considered in the literature. Firstly, the minimization of the determinant of the shape matrix of an ellipsoid is proposed. This is equivalent to the minimization of the square of the volume of the ellipsoid, but sometimes can lead to very narrow ellipsoids corresponding to very large uncertainties in some states [11]. Secondly, the trace criterion, which is equal to the sum of

S. Ben Chabane, C. Stoica Maniu and D. Dumur are with SUPELEC Systems Sciences (E3S) - Automatic Control Department, 3 rue Joliot Curie, 91192, Gif sur Yvette cedex, France (e-mail: \{ sofiane . benchabane; cristina.stoica; didier.dumur\}esupelec.fr).

T. Alamo and E.F. Camacho are with Department of Ingeniería de Sistemas y Automática, Universidad de Sevilla, Camino de los Descubrimientos, 41092 Sevilla, Spain (e-mail: a lamo@cartuja.us.es, eduardo@esi.us.es). squares of the half lengths of the axes of the ellipsoid, leads to better conditioned ellipsoids [11].

In addition, zonotopes are proposed to obtain a better estimation accuracy than ellipsoids. To minimize the size of the zonotopic estimation, several methods are proposed in the literature. In [14], a method based on the Singular Value Decomposition is used in order to obtain a zonotopic outer approximation of the intersection of the uncertain trajectory and the region which is consistent with the measured output. In [15], the minimization of the segments and the volume of the zonotope are used. The segments minimization method is faster but less accurate than the minimization of the volume of the zonotope. In [16], the minimization of the $P$-radius of the zonotope leads to a good trade-off between the rapidity of the segments minimization and the accuracy of the volume minimization of a zonotope.

This paper proposes an improved method which combines the advantages of the zonotopic set-membership state estimation (i.e. accuracy) and of the ellipsoidal set-membership estimation (i.e. reduced complexity). This is formulated as an optimization problem which starts with the zonotopic estimation and continues with the ellipsoidal estimation. A new criterion based on the $P$-radius of the zonotopic estimation is used to make the transition. In fact, when the $P$-radius of the zonotope is decreasing very slowly in the last iterations, an outer ellipsoidal approximation of the zonotope is considered to continue the state estimation with the ellipsoidal setmembership technique. This ellipsoid is obtained by scaling the ellipsoid related to the $P$-radius of the zonotope. This combined method (which is the novelty of this paper) leads to a less complex estimation than the zonotopic estimation based on the minimization of the $P$-radius and more accurate than the ellipsoidal estimation.

This paper is organized as follows. In Section II, the class of the uncertain systems used in this paper and the estimation problem are formulated. Section III and Section IV present the methods used in ellipsoidal state estimation and zonotopic state estimation, respectively. The next section presents the proposed method based on both zonotopes and ellipsoids to estimate the state. Section VI proposes an illustrative example which allows comparing the results obtained with ellipsoidal set-membership estimation techniques, zonotopic setmembership estimation and the proposed approach. Finally, some concluding remarks and perspectives are drawn.

\section{Notations and definitions}

An ellipsoid is defined by $\mathcal{E}(c, P)=\left\{x:(x-c)^{\top} P^{-1}(x-\right.$ $c) \leq 1\}$, where $c \in \mathbb{R}^{n}$ is the center of the ellipsoid and the matrix $P=P^{\top} \succ 0$ characterizes its shape and size. This 
ellipsoid is non-empty bounded (i.e. $\exists P^{-1}$ ).

A strip is defined by the following set $\mathcal{S}(y, d)=\left\{x \in \mathbb{R}^{n}\right.$ : $\left.\left|y-d^{\top} x\right| \leq 1\right\}$.

The unitary interval is defined by $\mathbf{B}=[-1,1]$.

The Minkovsky sum of two sets $\mathcal{A}$ and $\mathcal{B}$ is defined by $\mathcal{A} \oplus \mathcal{B}=\{a+b: a \in \mathcal{A}, b \in \mathcal{B}\}$.

A $m$-zonotope in $\mathbb{R}^{n}$ is defined by $\mathcal{Z}=\left\{x \in \mathbb{R}^{n}: x=\right.$ $\left.p+H z, z \in \mathbf{B}^{m}\right\}$, where the vector $p \in \mathbb{R}^{n}$ is the center of the zonotope, the matrix $H \in \mathbb{R}^{n \times m}$ represents the linear transformation and $\mathbf{B}^{m}$ is a box (interval vector) composed by $m$ unitary intervals.

Given a zonotope $\mathcal{Z}$, denote by $\mathcal{V}_{\mathcal{Z}}$ the set of its vertices.

The $P$-radius of a $m$-zonotope $\mathcal{Z}$ is a distance $d(x)=$ $\max _{x \in \mathcal{Z}}\|x-p\|_{P}^{2}$, with $P=P^{\top} \succ 0$.

\section{Problem StATEMENT}

Consider the following linear discrete-time invariant system with the state and measurement equations:

$$
\left\{\begin{array}{l}
x_{k+1}=A x_{k}+F \omega_{k} \\
y_{k}=c^{\top} x_{k}+\sigma v_{k}
\end{array}\right.
$$

where $x_{k} \in \mathbb{R}^{n}$ is the state vector, $y_{k} \in \mathbb{R}$ is the measurement output, $\omega_{k} \in \mathbb{R}^{n}$ and $v_{k} \in \mathbb{R}$ represent the state disturbances and the measurement noises, respectively. Here, $A, c, F$ and $\sigma$ have the appropriate dimensions, with the additional hypothesis that the pair $\left(c^{\top}, A\right)$ is detectable. It is assumed that the initial state $x_{0}$ belongs to the bounded set $\mathcal{X}_{0}$ and the perturbations and measurement noises are bounded by the compacts sets $\omega_{k} \in \mathcal{W}$ and $v_{k} \in \mathcal{V}$, respectively.

With these assumptions, using the set-membership theory and assuming that $x_{k} \in \hat{\mathcal{X}}_{k}$ (bounded set), the system state is contained in the set $\hat{\mathcal{X}}_{k+1}$, which is computed in two steps. The first is the prediction step, where the predicted state set $\overline{\mathcal{X}}_{k+1}$ is given by:

$$
\overline{\mathcal{X}}_{k+1}=A \hat{\mathcal{X}}_{k} \cup F \mathcal{W}
$$

The second is the correction step, where the guaranteed state set $\hat{\mathcal{X}}_{k+1}$ is given by:

$$
\overline{\mathcal{X}}_{k+1} \cap \mathcal{X}_{y_{k+1}} \subseteq \hat{\mathcal{X}}_{k+1}
$$

where

$$
\mathcal{X}_{y_{k+1}}=\left\{x_{k+1} \in \mathbb{R}^{n}:\left|c^{\top} x_{k+1}-y_{k+1}\right| \leq \sigma\right\}
$$

is the consistent state set using the output measurement $y_{k+1}$.

The aim is to find the optimal set $\hat{\mathcal{X}}_{k+1}$ that contains the state vector $x_{k+1}$. In the following, two methods to compute this set using ellipsoids and zonotopes are presented.

\section{ELLIPSOIDAL STATE ESTIMATION}

This section considers an ellipsoidal state estimation [11] for the system (1). The initial state vector $x_{0}$ belongs to the ellipsoid $\mathcal{E}_{0}\left(c_{0}, P_{0}\right), \mathcal{W}$ is the ellipsoid $\mathcal{E}\left(0, I_{2}\right)$ and $\mathcal{V}=\mathbf{B}$.

The size of an ellipsoid is measured by either its volume or by the sum of squares of the length of its semiaxes. The first is proportional to the square of the product of the length of its axes, which corresponds to the determinant criterion and the second corresponds to the trace criterion [11].

In the following, the two-step estimation procedure (prediction and correction) is addressed using the trace and the determinant criteria.

\section{A. Prediction step}

The prediction set, computed by considering ellipsoidal sets for $\overline{\mathcal{X}}_{k+1}, \hat{\mathcal{X}}_{k}$ and $\mathcal{W}$ in (2), is given by:

$$
\overline{\mathcal{E}}_{k+1}\left(c_{k+1}, P_{k+1}\right)=A \hat{\mathcal{E}}_{k}\left(c_{k}, P_{k}\right) \cup F \mathcal{E}\left(0, I_{2}\right)
$$

such that $x_{k}$ belongs to the guaranteed state set $\hat{\mathcal{E}}_{k}\left(c_{k}, P_{k}\right)$. Equation (5) can be rewritten as:

$$
\overline{\mathcal{E}}_{k+1}\left(c_{k+1}, P_{k+1}\right)=\hat{\mathcal{E}}_{k}\left(A c_{k}, A P_{k} A^{\top}\right) \cup \mathcal{E}\left(0, F F^{\top}\right)
$$

Applying the property of the sum of ellipsoids [11] means that there exists $\phi \in(0,1)$ such that:

$$
\left\{\begin{array}{l}
c_{k+1}=A c_{k} \\
P_{k+1}=\phi^{-1} P_{1}+(1-\phi)^{-1} P_{2}
\end{array}\right.
$$

with $P_{1}=A P_{k} A^{\top}$ and $P_{2}=F F^{\top}$.

The objective is to find $\phi$ which minimizes either the trace criterion:

$$
\phi^{*}=\arg _{0<\phi<1} \min \operatorname{tr}\left(P_{k+1}\right)
$$

or the determinant criterion:

$$
\phi^{*}=\arg _{0<\phi<1} \min \log \operatorname{det}\left(P_{k+1}\right)
$$

These two cases are detailed below.

1) Trace criterion: The advantage of this method consists in providing an explicit solution for $\phi^{*}$ (see [11] for the proof of concept):

$$
P_{k+1}=\left(\sqrt{\operatorname{tr}\left(P_{1}\right)}+\sqrt{\operatorname{tr}\left(P_{2}\right)}\right)\left(\frac{P_{1}}{\sqrt{\operatorname{tr}\left(P_{1}\right)}}+\frac{P_{2}}{\sqrt{\operatorname{tr}\left(P_{2}\right)}}\right)
$$

leading to the following optimal value:

$$
\phi_{i}^{*}=\left(\sqrt{\operatorname{tr}\left(P_{1}\right)}+\sqrt{\operatorname{tr}\left(P_{2}\right)}\right)^{-1} \sqrt{\operatorname{tr}\left(P_{i}\right)}
$$

2) Determinant criterion [11]: There is no explicit solution for this minimization problem. The recursive algorithm gives:

$$
P_{k+1}=\phi^{-1} P_{1}+(1-\phi)^{-1} P_{2}, \text { such that } \phi \in(0,1)
$$

The optimal value $\phi^{*}$ is obtained by solving a convex optimization problem of one dimension:

$$
\phi^{*}=\arg _{0<\phi<1} \min \log \operatorname{det}\left(\phi^{-1} P_{1}+(1-\phi)^{-1} P_{2}\right)
$$

Then, standard iterative methods for solving convex constrained optimization problems can be applied. 


\section{B. Correction step}

The goal of the correction step is to compute the intersection between the ellipsoid resulting after the prediction step and the strip representing the measured output. For this, the Outer Bounding Ellipsoid (OBE) algorithm [6] is used based on the minimal volume (determinant criterion) and minimal trace. In this step, the set $\hat{\mathcal{E}}_{k+1}\left(\hat{c}_{k+1}, \hat{P}_{k+1}\right)$ is found. This set is consistent with the measurement and it is computed by considering ellipsoidal sets for $\hat{\mathcal{X}}_{k+1}$ and $\overline{\mathcal{X}}_{k+1}$ in (3), i.e.:

$$
\overline{\mathcal{E}}_{k+1}\left(c_{k+1}, P_{k+1}\right) \cap \mathcal{X}_{y_{k+1}} \subseteq \hat{\mathcal{E}}_{k+1}\left(\hat{c}_{k+1}, \hat{P}_{k+1}\right)
$$

with the measurement consistent state set $\mathcal{X}_{y_{k+1}}=\left\{x_{k+1} \in\right.$ $\left.\mathbb{R}^{n}:\left(y_{k+1}^{\prime}-c^{\prime \top} x_{k+1}\right)^{2} \leq 1\right\}$, with $y_{k+1}^{\prime}=y_{k+1} \sigma$ and $c^{\prime}=$ $c \sigma$. From the intersection between an ellipsoid and a strip [6], it leads to:

$$
\begin{aligned}
& \hat{c}_{k+1}=c_{k+1}+\frac{\psi \delta}{1+\psi g} P_{k+1} c^{\prime} \\
& \hat{P}_{k+1}=\left(1+\psi-\frac{\psi \delta^{2}}{1+\psi g}\right)\left(P_{k+1}-\frac{\psi}{1+\psi g} P_{k+1} c^{\prime} c^{\prime \top} P_{k+1}\right)
\end{aligned}
$$

with $g=c^{\prime \top} P_{k+1} c^{\prime}, \delta=y_{k+1}^{\prime}-c^{\prime \top} c_{k+1}$ and $\psi \geq 0$.

The new ellipsoid contains the free parameter $\psi \geq 0$, which actually defines its position, size and orientation. Several criteria were developed to calculate this parameter. In the following, the determinant criterion and the trace criterion [6] will be presented.

1) Trace criterion [6]: Applying the fact that $\operatorname{tr}(M+$ $\left.u v^{\top}\right)=\operatorname{tr}(M)+v^{\top} u$ with $M=P_{k+1}, u=P_{k+1} c^{\prime}$ and $v=-\frac{\psi}{1+\psi g} P_{k+1} c^{\prime}$ to the trace of $\hat{P}_{k+1}$ in (15) leads to:

$$
\operatorname{tr}\left(\hat{P}_{k+1}\right)=\left(1+\psi-\frac{\psi \delta^{2}}{1+\psi g}\right)\left(\mu-\frac{\psi \gamma}{1+\psi g}\right)
$$

with $\mu=\operatorname{tr}\left(P_{k+1}\right)$ and $\gamma=c^{\prime \top} P_{k+1}^{2} c^{\prime}$.

The minimal trace ellipsoid is obtained by solving the following optimization problem:

$$
\psi^{*}=\arg _{\psi \geq 0} \min \operatorname{tr}\left(\hat{P}_{k+1}\right) .
$$

The optimal value of $\psi$ is the solution of:

$$
\frac{d \hat{P}_{k+1}(\psi)}{d \psi}=0
$$

As $\psi$ has to verify a condition of positivity $(\psi \geq 0)$ while satisfying (18), $\psi$ is the unique positive real root (Descartes's Rule of Signs [18]) of the following third degree polynomial:

$$
\frac{d \hat{P}_{k+1}(\psi)}{d \psi}=0 \Longleftrightarrow \psi^{3}+\beta_{1} \psi^{2}+\beta_{2} \psi+\beta_{3}=0
$$

with

$$
\left\{\begin{array}{l}
\beta_{1}=\frac{3}{g}>0 \\
\beta_{2}=\frac{g\left[\mu\left(1-\delta^{2}\right)-\gamma\right]+2\left[g \mu-\gamma\left(1-\delta^{2}\right)\right]}{g^{2}(g \mu-\gamma)} \\
\beta_{3}=\frac{\mu\left(1-\delta^{2}\right)-\gamma}{g^{2}(g \mu-\gamma)}
\end{array}\right.
$$

where $\beta_{3}$ and $\beta_{2}$ have the same sign.
2) Determinant criterion [6]: Applying the fact that $\operatorname{det}(\theta K)=\theta^{n} \operatorname{det}(K)$, with $\theta=1+\psi-\frac{\psi \delta^{2}}{1+\psi g} \in \mathbb{R}$ and $K \in \mathbb{R}^{n \times n}$ and the fact that $\operatorname{det}\left(I+u v^{\top}\right)=1+v^{\top} u$, with $u=P_{k+1} c^{\prime}$ and $v=-\frac{\psi}{1+\psi g} c^{\prime}$, then the determinant of $\hat{P}_{k+1}$ in (15) can be computed as follows:

$$
\operatorname{det}\left(\hat{P}_{k+1}\right)=\left(1+\psi-\frac{\psi \delta^{2}}{1+\psi g}\right)^{n} \frac{\operatorname{det}\left(P_{k+1}\right)}{1+\psi g}
$$

The minimal volume ellipsoid is obtained by solving the following optimization problem:

$$
\psi^{*}=\arg _{\psi \geq 0} \min h(\psi)
$$

with $h(\psi)=\left(1+\psi-\frac{\psi \delta^{2}}{1+\psi g}\right)^{n} /(1+\psi g)$.

A sufficient condition for $\psi$ is that:

$$
\frac{d h(\psi)}{d \psi}=0, \text { with } \psi \geq 0,
$$

which leads to the following solution (see [6] for more details):

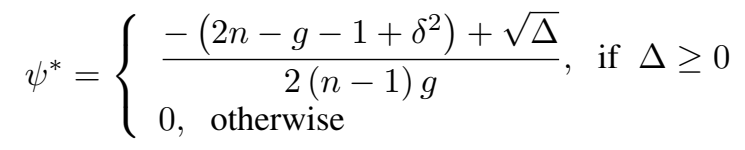

with $\Delta=\left(g-1-\delta^{2}\right)^{2}+4 \delta^{2}\left(\delta^{2}-1\right)$ and $n$ equal to the dimension of $x$. It can be noticed that $\psi^{*}=0$ means that the ellipsoid does not change $\left(\hat{\mathcal{E}}_{k+1}=\overline{\mathcal{E}}_{k+1}\right)$.

\section{ZONOTOPIC STATE ESTIMATION}

This section considers a zonotopic state estimation for the system (1). In this approach, it is assumed that the initial state $x_{0}$ belongs to the zonotope $\mathcal{Z}_{0}$, the perturbations are bounded by the box $\mathcal{W} \subseteq \mathbf{B}^{2}$ and the measurement noise is bounded by the unitary interval $\mathcal{V} \subseteq \mathbf{B}$. Suppose that the guaranteed state estimation set at time $k$ is a known zonotope:

$$
\hat{\mathcal{Z}}_{k}=\hat{p}_{k} \oplus \hat{H}_{k} \mathbf{B}^{r} \subset \mathbb{R}^{n}
$$

i.e. $\hat{\mathcal{X}}_{k}$ in (2) is equal to $\hat{\mathcal{Z}}_{k}$.

As shown in the previous sections, a similar two-step procedure (prediction and correction) is applied in order to compute the set containing the state.

\section{A. Prediction step}

The predicted state set $\overline{\mathcal{X}}_{k+1}=\overline{\mathcal{Z}}_{k+1}$ in (2) is determined as [16]:

$$
\overline{\mathcal{Z}}_{k+1}=A \hat{\mathcal{Z}}_{k} \oplus F \mathcal{W}=A \hat{p}_{k} \oplus\left[\begin{array}{ll}
\hat{H}_{k} & F
\end{array}\right] \mathbf{B}^{r+n} .
$$

Note that the union of two zonotopes is equal to the Minkovsky sum of these two zonotopes. 


\section{B. Correction step}

The guaranteed state estimation $\left(\hat{\mathcal{X}}_{k+1}=\hat{\mathcal{Z}}_{k+1}\right.$ in (3)) at time instant $k+1$ is the outer approximation of $\overline{\mathcal{Z}}_{k+1} \cap \mathcal{X}_{y_{k+1}}$. It is parametrized by the vector $\lambda$ (see [16] for more details), leading to the following family of zonotopes:

$$
\hat{\mathcal{Z}}_{k+1}(\lambda)=\hat{p}_{k+1}(\lambda) \oplus \hat{H}_{k+1}(\lambda) \mathbf{B}^{r+n+1}
$$

with

$$
\left\{\begin{array}{l}
\hat{p}_{k+1}(\lambda)=A \hat{p}_{k}+\lambda\left(y_{k+1}-c^{\top} A \hat{p}_{k}\right) \\
\hat{H}_{k+1}(\lambda)=\left[\begin{array}{lll}
\left(I-\lambda c^{\top}\right) A \hat{H}_{k} & \left(I-\lambda c^{\top}\right) F & \sigma \lambda
\end{array}\right]
\end{array}\right.
$$

The vector $\lambda \in \mathbb{R}^{n}$ is then determined in order to minimize the size of the zonotope $\hat{\mathcal{Z}}_{k+1}(\lambda)$.

In the literature, there are several methods for minimizing a zonotope based on its segments minimization [15], on its volume minimization [15] or on its $P$-radius minimization [16]. The first method offers a reduced complexity, while the second method leads to the most accurate result. The $P$-radius minimization method allows managing the trade-off between computational complexity and accuracy of the estimation. It is based on the minimization of the $P$-radius of the zonotope $\hat{\mathcal{Z}}_{k+1}(\lambda)$. The idea is to compute a matrix $P=P^{\top} \succeq 0$ and a vector $\lambda \in \mathbb{R}^{n}$ such that, at each iteration the $P$-radius of the zonotopic state estimation set is not increased.

Consider the $P$-radius of the state estimation set at time instant $k$ :

$$
L_{k}=\max _{x \in \hat{\mathcal{Z}}_{k}(\lambda)}\left\|x-\hat{p}_{k}\right\|_{P}^{2}=\max _{x \in \hat{\mathcal{Z}}_{k}(\lambda)}\left(x-\hat{p}_{k}\right)^{\top} P\left(x-\hat{p}_{k}\right)
$$

Note that the ellipsoid related to the $P$-radius of the zonotopic state estimation $\hat{\mathcal{Z}}_{k}$ is represented by:

$$
\mathcal{E}\left(\hat{p}_{k}, L_{k} P^{-1}\right)=\left\{x \in \mathbb{R}^{n}:\left\|x-\hat{p}_{k}\right\|_{P}^{2} \leq L_{k}\right\} .
$$

The non-increasing condition of the $P$-radius can be expressed as follows [16]:

$$
L_{k+1} \leq \beta L_{k}+\max _{\omega \in \mathbf{B}^{n}}\|F \omega\|_{2}^{2}+\sigma^{2}
$$

with $\beta \in(0,1)$.

Expression (31) is equivalent to:

$$
\max _{z \in \mathbf{B}^{r+n+1}}\left\|\hat{H}_{k+1} \hat{z}\right\|_{P}^{2} \leq \max _{z \in \mathbf{B}^{r}} \beta\left\|\hat{H}_{k} z\right\|_{P}^{2}+\max _{\omega \in \mathbf{B}^{n}}\|F \omega\|_{2}^{2}+\sigma^{2}
$$

with $\hat{z}=\left[z^{\top} \omega^{\top} \eta\right]^{\top} \in \mathbf{B}^{r+n+1}, z \in \mathbf{B}^{r}, \omega \in \mathbf{B}^{n}, \eta \in \mathbf{B}$ and $\beta \in(0,1)$. A sufficient condition for the inequality (32) is given in [16]. This consists in finding the smallest value of $\beta \in(0,1)$ (for instance via the bisection algorithm) such that the following optimization is feasible:

$$
\max _{\tau, P, \beta, Y} \tau
$$

subject to

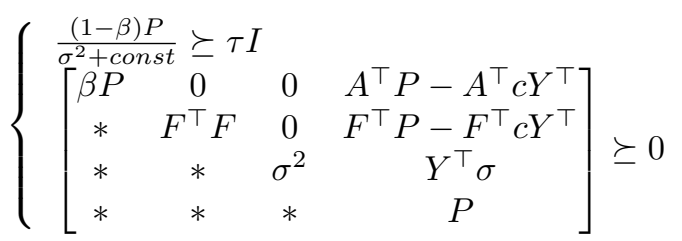

with const $=\max _{\omega \in \mathbf{B}^{n}}\|F \omega\|_{2}^{2}$. The decision variables are $\tau>0$, $P \in \mathbb{R}^{n \times n}, \beta \in(0,1)$ and $Y=P \lambda \in \mathbb{R}^{n}$.

The optimization problem (33) is computed off-line which leads to a small computation complexity compared to the zonotope volume minimization [16] for a similar accuracy. However this $P$-radius based problem is still enough complex compared to the ellipsoidal minimization. This motivates the improved approach proposed in the next section.

\section{PROPOSED APPROACH}

This section proposes an improved method which takes advantage of the good estimation accuracy obtained via the zonotopic minimization and the low complexity proven by the ellipsoidal minimization.

In fact, when the $P$-radius is decreasing slowly during the last iterations of the zonotopic estimation, (i.e. the solution is close enough to an optimum), we propose to outer approximate the zonotopic estimation set by an ellipsoid and to continue the procedure via the ellipsoidal set-membership estimation. This leads to gain in rapidity of the estimation, while keeping an acceptable level of accuracy. The design of this outer ellipsoidal approximation of the zonotopic state set is further detailed. Computing the zonotopic state estimation set via the minimization of the $P$-radius (i.e. solving the optimization problem (33)) allows computing the $P$-radius of the zonotope and its associated ellipsoid. The ellipsoid related to the $P$ radius is usually an inner approximation of the zonotope, with the same center and direction as the zonotope (e.g. the blue dashed ellipsoid in Fig. (1)). In order to obtain an outer ellipsoid that will outer approximate the zonotope, the ellipsoid related to the $P$-radius will be scaled by a scalar $\alpha$ (leading to the red ellipsoid in Fig. (1)). The parameter $\alpha \in(0,1)$ has to be found in order to minimize the size of the ellipsoid $\hat{\mathcal{E}}_{k}=\left\{x_{k} \in \mathbb{R}^{n}:\left(x_{k}-\hat{p}_{k}\right)^{\top} \alpha\left(L_{k} P^{-1}\right)^{-1}\left(x_{k}-\hat{p}_{k}\right) \leq 1\right\}$, where $\hat{p}_{k}$ is the center of the zonotope (computed using expression (28)) to be outer bounded and $L_{k}$ its $P$-radius. This can be formulated as a optimization problem that has to be verified for all the vertices of the zonotope $\hat{\mathcal{Z}}_{k}$ :

$\max \alpha$

subject to

$$
\left\{\begin{array}{l}
0<\alpha<1 \\
\left(x_{k}-\hat{p}_{k}\right)^{\top} \alpha P L_{k}^{-1}\left(x_{k}-\hat{p}_{k}\right) \leq 1, \quad \forall x_{k} \in \mathcal{V}_{\hat{\mathcal{Z}}_{k}}
\end{array}\right.
$$

To compute $\alpha$, equation (34) has to be verified for all the vertices $\mathcal{V}_{\hat{\mathcal{Z}}_{k}}$ of the zonotope $\hat{\mathcal{Z}}_{k}$ which requires a large computation time. It is suitable to find a method reducing the number of vertices to be checked. Thus, let us denote by $\bar{x}_{\hat{\mathcal{Z}}_{k}}$ the furthest vertex from the center of the zonotope. Then, the following proposition avoids the vertex enumeration when solving the optimization problem (34):

Proposition 1: Consider a matrix $P=P^{\top} \succ 0 \in \mathbb{R}^{n \times n}$, a scalar $\alpha \in(0,1)$, the state vector $x_{k} \in \mathbb{R}^{n}$ belonging to the zonotope $\hat{\mathcal{Z}}_{k}=\hat{p}_{k} \oplus \hat{H}_{k} \mathbf{B}^{m}$, with the associated $P$-radius $L_{k}>0$ and the furthest vertex $\bar{x}_{\mathcal{Z}_{k}} \in \mathbb{R}^{n}$ with respect to its center. If $\left(\bar{x}_{\hat{\mathcal{z}}_{k}}-\hat{p}_{k}\right)^{\top} \alpha P L_{k}^{-1}\left(\bar{x}_{\hat{\mathcal{Z}}_{k}}-\hat{p}_{k}\right) \leq 1$, then $\left(x_{k}-\right.$ $\left.\hat{p}_{k}\right)^{\top} \alpha P L_{k}^{-1}\left(x_{k}-\hat{p}_{k}\right) \leq 1, \quad \forall x \in \mathcal{V}_{\hat{\mathcal{Z}}_{k}}$. 


\section{Proof:}

Expression (29) can be formulated as follows:

$$
\left(\bar{x}_{\hat{\mathcal{Z}}_{k}}-\hat{p}_{k}\right)^{\top} P\left(\bar{x}_{\hat{\mathcal{Z}}_{k}}-\hat{p}_{k}\right)=L_{k},
$$

with $\bar{x}_{\hat{\mathcal{Z}}_{k}}$ the furthest vertex from the center of $\hat{\mathcal{Z}}_{k}$. This means that:

$\left(x_{k}-\hat{p}_{k}\right)^{\top} P\left(x_{k}-\hat{p}_{k}\right) \leq\left(\bar{x}_{\hat{\mathcal{Z}}_{k}}-\hat{p}_{k}\right)^{\top} P\left(\bar{x}_{\hat{\mathcal{Z}}_{k}}-\hat{p}_{k}\right), \quad \forall x_{k} \in \mathcal{V}_{\hat{\mathcal{Z}}_{k}}$.

Assume that $\exists \alpha \in(0,1)$ such that:

$$
\left(\bar{x}_{\hat{\mathcal{Z}}_{k}}-\hat{p}_{k}\right)^{\top} \alpha P L_{k}^{-1}\left(\bar{x}_{\hat{\mathcal{z}}_{k}}-\hat{p}_{k}\right) \leq 1 .
$$

This inequality can be rewritten as:

$$
\left(\bar{x}_{\hat{\mathcal{Z}}_{k}}-\hat{p}_{k}\right)^{\top} P\left(\bar{x}_{\hat{\mathcal{Z}}_{k}}-\hat{p}_{k}\right) \leq \frac{L_{k}}{\alpha} .
$$

From (36) and (38) it is inferred that:

$$
\left(x_{k}-\hat{p}_{k}\right)^{\top} P\left(x_{k}-\hat{p}_{k}\right) \leq \frac{L_{k}}{\alpha}, \quad \forall x_{k} \in \mathcal{V}_{\hat{\mathcal{Z}}_{k}}
$$

which is equivalent to:

$$
\left(x_{k}-\hat{p}_{k}\right)^{\top} \alpha P L_{k}^{-1}\left(x_{k}-\hat{p}_{k}\right) \leq 1, \quad \forall x \in \mathcal{V}_{\hat{\mathcal{Z}}_{k}} .
$$

This proposition avoids solving the optimization problem (34) for all the vertices of the zonotope (only the furthest vertex will be now considered in (34)) and gives a reduced computation time.

The following algorithm formulates the proposed method.

\section{Algorithm 1:}

\section{Inputs:}

$N$ : length of the simulation horizon;

$\epsilon$ : desired level of accuracy of the $P$-radius;

$x_{0}$ : initial state vector;

$\hat{\mathcal{Z}}_{0}$ : initial state set;

$l$ : length of the horizon of slow variation of the $P$-radius.

\section{Outputs:}

test $=1$;

for $k=1: N$

if $\quad\left(\left|L_{k}-L_{k-l}\right| \geq \epsilon\right.$ and test $\left.=1\right)$ or $(k \leq l)$ then Compute the zonotope estimation $\hat{\mathcal{Z}}_{k}$ and its $P$-radius $L_{k}$;

test $=1$

else if $\left|L_{k}-L_{k-l}\right| \leq \epsilon$ and test $=1$ then

Compute the scaled ellipsoid $\hat{\mathcal{E}}_{k}$;

$$
\text { test }=0 \text {; }
$$

else

Compute the ellipsoid estimation $\hat{\mathcal{E}}_{k}$ via the trace minimization estimation; test $=0$;

end

Collect the measurement $y_{k+1}$; end

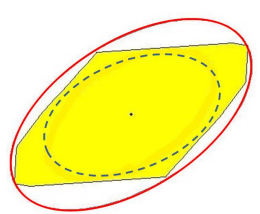

Fig. 1. Ellispoidal outer-bounding approximation of a zonotope

It means that if the $P$-radius does not change after $l$ iterations, a transition to the ellipsoidal estimation is made and the algorithm continues with the ellipsoidal estimation to end of the simulation. In addition, the scalar $\epsilon$ is fixing the speed of transition to the ellipsoidal estimation method (i.e. it is inversely proportional to the speed of this transition). At the moment of transition, the ellipsoidal outer approximation is chosen as an outer bound of the zonotopic estimation with the same center and direction (given by the $P$ matrix) as the ellipsoid related to the $P$-radius of the zonotopic estimation. An illustrative example showing the performance of the proposed approach is presented in the next section.

\section{ILLUSTRATIVE EXAMPLE}

Consider the following linear discrete-time invariant system:

$$
\left\{\begin{array}{l}
x_{k+1}=\left[\begin{array}{cc}
1 & 1 \\
0 & 0.8
\end{array}\right] x_{k}+\left[\begin{array}{c}
-0.24 \\
0.04
\end{array}\right] \omega_{k} \\
y_{k}=\left[\begin{array}{ll}
-2 & 1
\end{array}\right] x_{k}+0.4 v_{k}
\end{array}\right.
$$

with $\left\|v_{k}\right\|_{\infty} \leq 1,\left\|\omega_{k}\right\|_{\infty} \leq 1$. The initial state belongs to the box $3 \mathbf{B}^{2}$. The order of the zonotope is limited to $m \leq 20$ in order to have a fast simulation.

Figures 2 and 3 illustrate the bounds of $x_{1}$ and $x_{2}$ after 120 iterations obtained with the three estimation methods: the $P$ radius based zonotopic estimation, the trace-based ellipsoidal estimation and the proposed method.

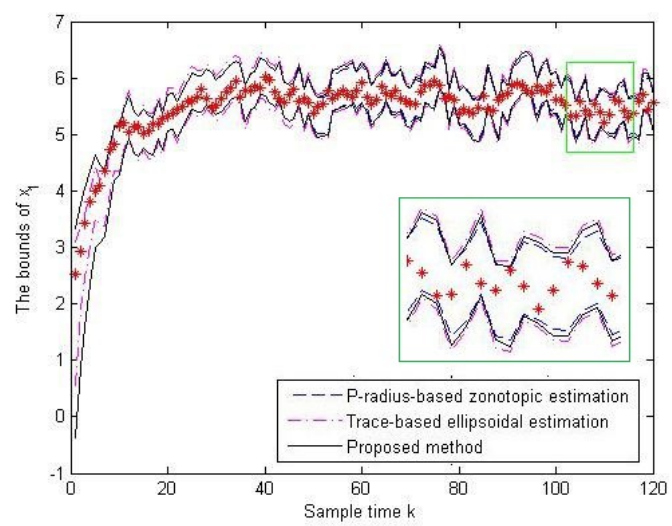

Fig. 2. Bounds of $x_{1}$ using the three methods

The magenta dashdot lines are obtained by the ellipsoidal estimation using the trace minimization criterion. The blue dashed lines are obtained by the zonotopic estimation based on the $P$-radius minimization and the black solid lines are 


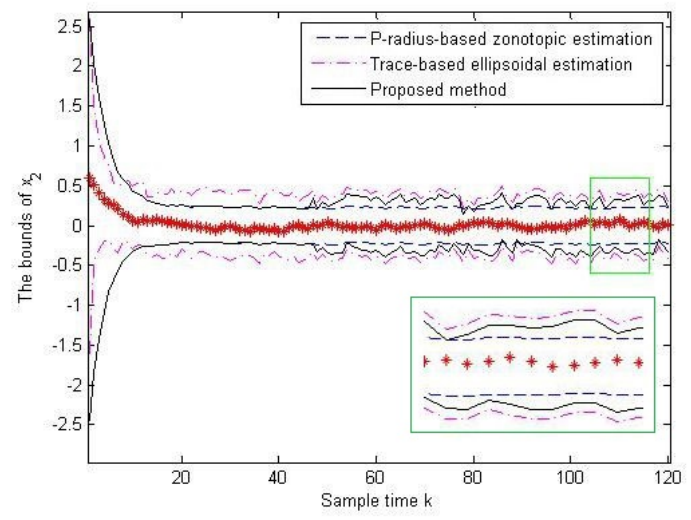

Fig. 3. Bounds of $x_{2}$ using the three methods

obtained using the proposed method. The red stars represent the real state of the system, which is inside the estimated sets.

The computation time after $N=120$ iterations is $0.27 s$ for $P$-radius-based zonotopic estimation, $0.08 s$ for the trace-based ellipsoidal estimation and $0.15 \mathrm{~s}$ for the proposed method, which is a good trade-off. This results are obtained using an Intel Core Duo E8500 3.16GHz. The tuning parameters used in Algorithm $l$ are: $\epsilon=10^{-5}$ and $l=5$.

Figure 4 compares the volume of the state estimation sets obtained by the considered approaches. The minimal volume is obtained by the $P$-radius-based method, while with the proposed method the volume is the same until just before the transition and it does not degrade too much after the transition. The proposed method permits to gain $45 \%$ in computation time compared to the $P$-radius-based zonotopic estimation with a degradation of the volume of $36 \%$ compared to the $P$ radius-based zonotopic estimation, which remains reasonable. This confirms the objective of the proposed algorithm offering good accuracy compared to the ellipsoidal estimation, while reducing the complexity compared to the zonotopic estimation.

\section{CONCLUSION}

The aim of this paper is twofold: comparing zonotopic and ellipsoidal set-membership estimation techniques, and proposing a new approach allowing a trade-off between zonotopic and ellipsoidal estimation approaches.

An improved approach for guaranteed set-membership state estimation based on zonotopes and ellipsoids for linear systems with bounded perturbations and measurement noise has been proposed. It starts with the $P$-radius-based zonotopic state estimation and continues with the ellipsoidal state estimation. A new criterion based on the P-radius is proposed in order to make the transition from the zonotopic estimation to the ellipsoidal estimation. This method offers a good accuracy compared to the ellipsoidal estimation with reduced complexity compared to the zonotopic estimation. An example has been provided to motivate and clarify the choice of this method. Current work consists in applying the proposed method to fault detection and fault tolerant control purposes.

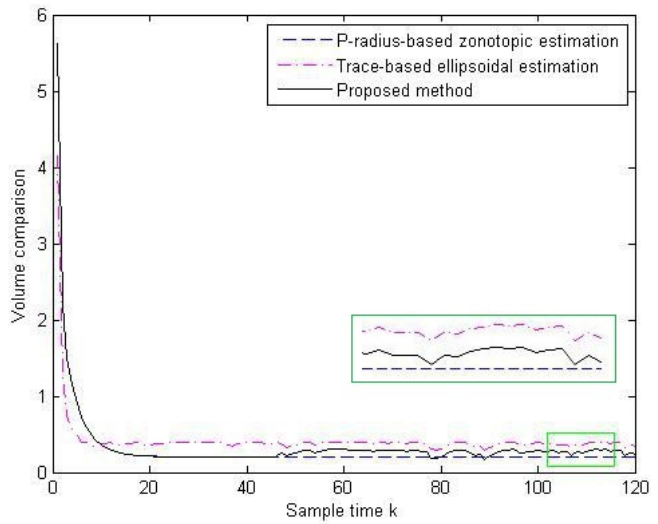

Fig. 4. Comparison of the volume of state estimation sets

\section{REFERENCES}

[1] R. E. Kalman, "A new approach to linear filtering and prediction problems," Transactions of the ASME-Journal of Basic Engineering, vol. 82, no. Series D, pp. 35-45, 1960.

[2] F. C. Schweppe, "Recursive state estimation: Unknown but bounded errors and system inputs," IEEE Trans. Automat. Contr., vol. 13(1), pp. 22-28, 1968.

[3] E. Fogel, "System identification via membership set constraints with energy constrained noise," IEEE Trans. Automat. Contr., vol. AC-24, p. 752, 1979.

[4] D. P. Bertsekas and I. B. Rhodes, "Recursive state estimation for a setmembership description of uncertainty," IEEE Trans. Automat. Contr., vol. 16(2), pp. 117-128, 1971.

[5] H. S. Wistenhausen, "Sets of possible states of linear systems given perturbed observations," IEEE Trans. Automat. Contr., vol. 13(5), pp. 556-558, 1968.

[6] E. Fogel and Y. F. Huang, "On the value of information in system identification-bounded noise case," Automatica, vol. 18, pp. 229-238, 1982.

[7] F. L. Chernousko and D. Y. Rokityanskii, "Ellipsoidal bounds on reachable sets of dynamical systems with matrices subjected to uncertain perturbations," Journal of Optimization Theory and Applications, vol. 104, pp. 1-19, 2000.

[8] E. Walter and H. Piet-Lahanier, "Exact recursive polyhedral description of the feasible parameter set for bounded-error models," IEEE Trans. Automat. Contr., vol. 34(8), pp. 911-915, 1989.

[9] L. Chisci, A. Garulli, and G. Zappa, "Recursive state bounding by parallelotopes," Automatica, vol. 32, pp. 1049-1055, 1996.

[10] A. Kurzhanski and I. Vályi, Ellipsoidal calculus for estimation and control. Birkhaüser Boston, 1996.

[11] C. Durieu, E. Walter, and B. Polyak, "Multi-input multi-output ellipsoidal state bounding," Journal of optimization theory and applications, vol. 111(2), pp. 273-303, 2001.

[12] B. Polyak, S. A. Nazin, C. Durieu, and E. Walter, "Ellipsoidal parameter or state estimation under model uncertainty," Automatica, vol. 40, pp. 1171-1179, 2004.

[13] V. Puig, P. Cugueró, and J. Quevedo, "Worst-case estimation and simulation of uncertain discrete-time systems using zonotopes," in Proc. of European Control Conference, Portugal, 2001.

[14] C. Combastel, "A state bounding observer based on zonotopes," in Proc. of European Control Conference, Cambridge, UK, 2003.

[15] T. Alamo, J. M. Bravo, and E. F. Camacho, "Guaranteed state estimation by zonotopes," Automatica, vol. 41, pp. 1035-1043, 2005.

[16] V. T. H. Le, C. Stoica, T. Alamo, E. F. Camacho, and D. Dumur, "Zonotopic guaranteed state estimation for uncertain systems," Automatica, vol. 49(1), pp. 3418-3424, 2013.

[17] B. Polyak, S. A. Nazin, C. Durieu, and E. Walter, "Ellipsoidal estimation under model uncertainty," in Proc. of the 15th World Congress IFAC, Barcelona, Spain, 2002.

[18] A. A. Albert, "An inductive proof of Descartes's rule of signs," American Mathematical Monthly, vol. 50(3), pp. 178-180, 1943. 